\title{
Characterization of single-crystal synthetic diamond for multi-watt continuous-wave Raman lasers
}

\author{
Vasili G. Savitski, Ian Friel, Jennifer E. Hastie, Member, IEEE, Martin D. Dawson, Fellow, IEEE, \\ David Burns, Alan J. Kemp, Member, IEEE
}

\begin{abstract}
A continuous-wave diamond Raman laser is demonstrated with an output power of $5.1 \mathrm{~W}$ at $1240 \mathrm{~nm}$. This Raman laser is intracavity pumped by a side-pumped Nd:YLF rod laser: a 43-fold brightness enhancement between the Nd:YLF and diamond Raman lasers is observed, with the $\mathbf{M}^{2}$ beam propagation factor of the diamond Raman laser measured to be $<1.2$. Although higher output powers are demonstrated in a similar configuration using $\mathrm{KGW}$ as the Raman laser material (6.1 W), the brightness enhancement is much lower ( 2.5 fold) due the poorer beam quality of the KGW Raman laser $\left(M^{2}<6\right)$. The Raman gain coefficient of single-crystal synthetic diamond at a pump wavelength of $1064 \mathrm{~nm}$ is also measured: a maximum value of $21 \pm 2 \mathrm{~cm} / \mathrm{GW}$ is returned compared to $5.7 \pm 0.5 \mathrm{~cm} / \mathrm{GW}$ for KGW at the same wavelength.
\end{abstract}

Index Terms-Raman gain, Raman laser, Raman scattering, Solid lasers

\section{INTRODUCTION}

$\mathrm{S}$ olid-state lasers based on doped dielectric crystals are capable of unrivalled performance: from ultrashort pulses to kilowatt output powers; from multi-joule pulses to hertzlevel linewidths. The output wavelength of a typical solidstate laser, however, is dictated by the electronic transitions of the dopant ion with very limited potential to engineer this to meet the requirements of a particular application. For this reason there is continuing interest in non-linear frequency conversion. The most flexible technique is the optical parametric oscillator [1]. Tuning over hundreds of nanometres based on a fixed wavelength pump laser is possible. However, the requirement to phasematch the pump and generated waves means that optical parametric oscillators can be relatively complicated, particular for continuous-wave operation.

An alternative approach - and the focus of considerable recent research - is the crystalline Raman laser [2-4]. This technique uses stimulated Raman scattering [5] to shift the

Manuscript received 13 October 2011. This work was funded by the UK EPSRC under grant number EP/E00014X/1.

Vasili G. Savitski, Jennifer E. Hastie, Martin D. Dawson, David Burns, and Alan J. Kemp are with the Institute of Photonics, University of Strathclyde, SUPA, 106 Rottenrow, Glasgow G4 0NW, UK (corresponding author phone: 44-141-548-3447; fax: 44-141-552-1575; e-mail: vasili.savitski@strath.ac.uk). Ian Friel is with Element Six Ltd., King's Ride Park, Ascot, SL5 8BP, UK. wavelength of the input laser. The disadvantage of this approach is the fixed frequency shift; the advantage is the simplicity that results from the removal of the phasematching constraints associated with optical parametric oscillators. Raman scattering is a $\chi^{3}$ nonlinear effect and therefore high intensities from pulsed pump lasers are typically used to achieve efficient conversion. In 2005, however, continuouswave solid-state Raman lasers were reported based on placing the Raman-active crystal inside the cavity of a modest diodepumped solid-state laser [6, 7]. This approach - an intracavity-pumped Raman laser - has been widely investigated as a means to shift the wavelength of established continuous-wave neodymium lasers. Fan et al. have reported continuous-wave output powers of $3.4 \mathrm{~W}$ at the first Stokes wavelength [8]. Lee et al. have demonstrated even higher continuous-wave powers in the visible by intracavity frequency conversion: $4.3 \mathrm{~W}$ at the second harmonic of the first Stokes wavelength [9] and up to $5.3 \mathrm{~W}$ via sum frequency mixing of the Stokes and fundamental fields [10]. Using diamond as the Raman laser material, the highest continuouswave output power achieved to date is $1.6 \mathrm{~W}$ at the first Stokes wavelength [11].

It is also possible to build continuous-wave Raman lasers based on an external cavity, as demonstrated by Brassuer et al. using hydrogen gas as the Raman material [12], Grabtchikov et al. using potassium gadolinium tungstate $\left(\mathrm{KGd}\left(\mathrm{WO}_{4}\right)_{2}-\right.$ known as $\mathrm{KGW}$ ) [13], and recently at higher average powers by Kitzler et al using diamond [14]. In quasi continuous-wave operation, Kitzler et al. demonstrated impressive on-time output powers of $7.5 \mathrm{~W}$ in $6.5 \mathrm{~ms}$ pulses. The duty cycle was $16.5 \%$ giving average powers of $1.2 \mathrm{~W}$.

The inelastic nature of Raman scattering means that heat is of necessity deposited into the Raman laser material - in contrast to optical parametric processes. As a result, intracavity Raman lasers contain two thermal lenses - one in the conventional laser material and one within the Raman material. The interplay of these lenses complicates the engineering of higher power continuous-wave Raman lasers [3]. This is compounded by the low thermal conductivity of common Raman laser crystals. As a result there is considerable interest in the use of diamond as a Raman laser material, both in pulsed [15-17] and continuous-wave formats 
TABLE I

COMPARISON OF THE THERMAL, MECHANICAL AND OPTICAL PROPERTIES OF DIAMOND AND KGW RELEVANT TO RAMAN LASERS

\begin{tabular}{llll}
\hline \hline & Diamond & KGW & Refs \\
\hline Thermal Conductivity, $k(\mathrm{~W} / \mathrm{mK})$ & 2000 & $3.8^{*}$ & {$[21,22]$} \\
$\begin{array}{l}\text { Thermal Expansion, } \alpha_{t h}\left(\mathrm{x} 10^{-6} \mathrm{~K}^{-1}\right) \\
\begin{array}{l}\text { Thermo-Optic Coefficient, } d n / d T \\
\left(\mathrm{x} 10^{-6} \mathrm{~K}^{-1}\right)\end{array}\end{array}$ & 1.0 & $17.4^{*}$ & {$[21,23]$} \\
$\begin{array}{l}\text { Typical Length of Crystal, } L(\mathrm{~mm}) \\
\begin{array}{l}\text { Max. Raman Gain Coeff., } g \text { at } \\
1064 \mathrm{~nm} \text { fundamental, }(\mathrm{cm} / \mathrm{GW})\end{array}\end{array}$ & 6 & $-17.3^{*}$ & {$[21,23]$} \\
\begin{tabular}{l} 
Raman Shift $\left(\mathrm{cm}^{-1}\right)$ \\
\hline \hline
\end{tabular}
\end{tabular}

* Property not isotropic - the maximum value is given for the purposes of comparison

$[11,18]$. This work is motivated by the exceptional thermal properties of diamond [19] and enabled by the recent development of high optical quality synthetic diamond grown by chemical vapour deposition [11,20,21].

In this paper, modern synthetic single-crystal diamond will be compared with KGW, a representative conventional Raman laser crystal. The materials properties of diamond and $\mathrm{KGW}$ will be discussed in the next section. Section 3 will then describe the samples used in this study before comparative pump-probe measurements of Raman gain, calorimetric measurements of absorption and Raman laser experiments are described in sections 4,5 and 6 .

\section{Properties OF Diamond AND KGW}

Until recently, the use of diamond in solid-state lasers was restricted by considerations of cost and material quality [2527] - in particular birefringence and absorption. Recent developments in chemical vapour deposition growth have led to material with low birefringence and low absorption through the minimisation of the dislocation density and nitrogen impurities respectively [20,21]. This has made the intracavity use of diamond in solid-state lasers more practical - as a heat spreader [28, 29] and in Raman lasers [11, 15-18].

The properties of diamond relevant to Raman lasers are set out in Table 1 and compared to a conventional material, KGW. The advantage of high optical quality synthetic diamond is clear in terms of thermal conductivity -600 fold higher than KGW - and Raman gain coefficient - four fold higher. These properties corresponds to the potential for much lower thermal lensing per unit Raman laser output power despite the shorter lengths of diamond available. Drawing on the work of Pask [2], Lubeigt et al [18] proposed a Raman laser figure of merit to characterise this. For diamond, this figure of merit is over two orders of magnitude higher than for $\mathrm{KGW}$ based on typically available lengths. Such advantages, coupled to the recent improvements in the optical quality of synthetic single crystal diamond $[11,20,21]$ have motivated the recent interest in diamond for Raman lasers. In this paper the properties of diamond will be experimentally assessed in the context of continuous-wave intracavity Raman lasers, using $\mathrm{KGW}$ as a standard for comparison. In the next section, the samples used experimentally will briefly be described

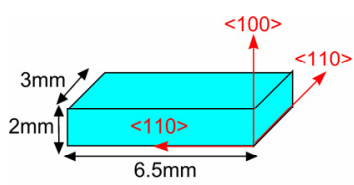

(a) Diamond Sample A

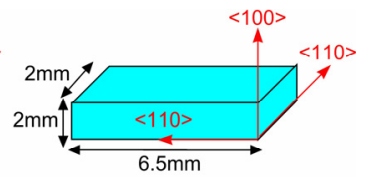

(b) Diamond Sample B $\mathrm{Nm}$

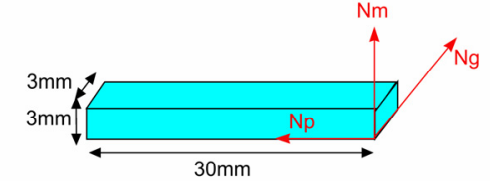

(c) KGW

Fig. 1: Orientation and dimensions of the samples examined: (a) diamond sample A; (b) diamond sample B; and (c) the KGW sample. (Diagrams are not to scale.)
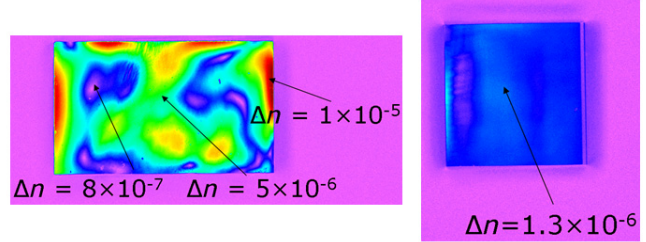

(a) Diamond Sample A

(b) Diamond Sample B

Fig. 2: Maps of the sine of phase retardation $\delta(|\sin (\delta)|=2 \pi(\Delta n) L / \lambda$,

where $\Delta n$ is the birefringence, $L$ - sample length, $\lambda$ - wavelength) of diamond samples $\mathrm{A}$ (a) and $\mathrm{B}$ (b). The associated values of $\Delta n$ are shown for particular locations. (Diagrams are not to scale.)

before the experimental results are discussed in subsequent sections.

\section{SYNTHETIC DIAMOND AND KGW SAMPLES}

The single crystal diamond samples were grown homoepitaxially by Element Six Ltd using microwave plasma assisted chemical vapour deposition. Growth was along the direction labeled $\langle 100\rangle$ in Fig. 1. The cut and polished samples were $6.5 \mathrm{~mm}$ long cuboids cut for light propagation along a $<110>$ axis (Fig. 1 (a) and (b)). The end faces of sample A were antireflection (AR) coated for 1 and $1.2 \mu \mathrm{m}$ (reflectivity $\mathrm{R}<0.15 \%$ ); sample B was left uncoated. Sample A was used for laser experiments and sample B for the Raman gain measurements.

Diamond sample A was grown specifically for low nitrogen content and hence low absorption loss. Sample B was grown to have ultra-low birefringence and contained $\sim 20 \mathrm{ppb}$ of single substitutional nitrogen. The birefringence $(\Delta n)$ along the direction of propagation (measured using the metripol technique at the wavelength $\lambda$ of $550 \mathrm{~nm}$ for light propagating along the $L=6.5 \mathrm{~mm}$ dimension of the crystal) varied from $1 \times 10^{-5}$ to $8 \times 10^{-7}$ for sample $\mathrm{A}$ and was measured to be $\sim 1.3 \times 10^{-6}$ for sample B (Fig. 2).

The $\mathrm{KGd}\left(\mathrm{WO}_{4}\right)_{2}$ crystal (KGW) was $30 \mathrm{~mm}$ in length (Fig. 1 (c)). It was supplied by Altechna Ltd. The end faces of the crystal were anti-reflection coated for 1 and $1.15 \mu \mathrm{m}$ $(\mathrm{R}<0.1 \%)$. The crystal was cut for light propagation along its $N_{p}$ axis. The following sections describe measurements of Raman gain, absorption and Raman laser performance using these crystals. 


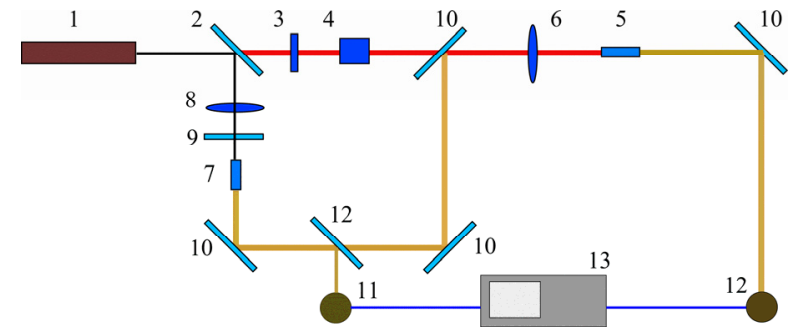

Fig. 3: Schematic diagram of the pump-probe set-up used for measurements of Raman gain. (1) Nd:YAG laser; (2) 50/50 beam splitter; (3) half-wavelength plate; (4) Glan-Taylor prism polariser; (5) sample under study; (6), (8) lenses; (7) sample generating the probe beam; (9), (10) dichroic mirrors; (11), (12) photodetectors; (13) digital oscilloscope.

\section{PUMP-PROBE RAMAN GAIN MEASUREMENTS}

The Raman gain in the KGW and diamond crystals was measured using a standard pump-probe technique [30-32]. The experimental set-up is shown in Fig. 3. The pump laser (1) was an actively Q-switched Nd:YAG laser emitting at $1064 \mathrm{~nm}$ and producing pulses with a maximum energy of 100 $\mathrm{mJ}$ (Continuum Minilite II). These pulses were split at the $50 / 50$ beam splitter (2). The part transmitted through the beam splitter was used to pump the sample under test. Having been attenuated using a half-wavelength plate (3) and a GlanTaylor prism polariser (4), the pump beam was focused into the sample (5) using a lens (6) with the focal length of 300 $\mathrm{mm}$.

The part of the laser beam reflected by beam splitter (2) was focused by lens (8) into a second KGW or diamond crystal (7) (depending on the material under test), generating a probe beam at the first Stokes wavelength. The dichroic mirror (9, highly transmissive at $1064 \mathrm{~nm}$ and highly reflective at 1150 $1250 \mathrm{~nm}$ ) and the uncoated back surface of the crystal formed a Raman laser cavity to produce the probe emission. The residual pump at $1064 \mathrm{~nm}$ was then filtered out using dichroic mirrors (10, highly transmissive at $1064 \mathrm{~nm}$ and highly reflective at $1150-1250 \mathrm{~nm}$ ). A small part of the probe emission was reflected onto a photo detector (11) by a beam splitter (12). The rest of the probe emission was focused into the sample under study (5) by lens (6). After reflection from the dichroic mirror $(10)$ the amplified probe emission reached a photo detector (12). The signals from detectors (11) and (12) were analysed using a digital oscilloscope (13). Attenuation was used to ensure that the ratio of the signals from detector (11) and detector (12) was equal to one when the sample (5) was not pumped.

The Raman gain can be extracted from the ratio of the relative intensity of the probe pulse after and before passing through the pumped sample. To calculate the Raman gain coefficient, $g$, the following equation was used [32]:

$g=\frac{E_{S}-E_{S 0}}{E_{S 0} E_{p} L}\left(\omega_{p}^{2}+\omega_{S}^{2}\right) \sqrt{\tau_{p}^{2}+\tau_{S}^{2}}\left(\frac{\pi}{2}\right)^{3 / 2}$

where $E_{S}\left(E_{S 0}\right)$ is the output (input) probe pulse energy, $E_{P}$ is

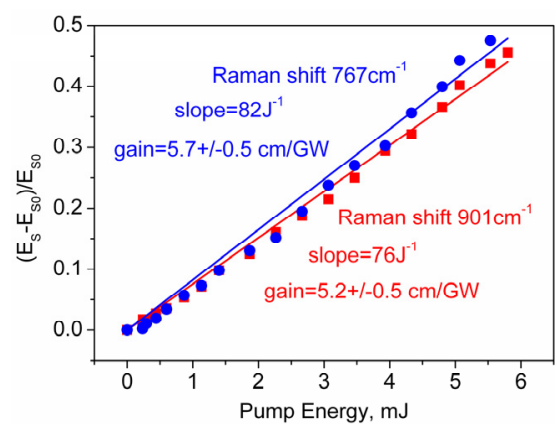

Fig. 4: Raman gain coefficient in KGW measured for the Raman shifts of 767 and $901 \mathrm{~cm}^{-1}$.

TABLE II

RAMAN GAIN IN KGW UNDER $1.06 \mu$ M PUMPING FOR RAMAN FREQUENCY SHIFTS OF 767 AND $901 \mathrm{CM}^{-1}$

\begin{tabular}{ccc}
\hline \hline \multicolumn{2}{c}{ Raman Gain Coefficient $(\mathrm{cm} / \mathrm{GW})$} & Ref. \\
\cline { 1 - 2 } $767 \mathrm{~cm}^{-1}$ shift & $901 \mathrm{~cm}^{-1}$ shift & {$[33]$} \\
6 & 6 & {$[24]$} \\
6 & 6 & {$[22]$} \\
4.4 & 6 & {$[34]$} \\
$5.7 \pm 0.5$ & 4.8 & This report \\
\hline \hline
\end{tabular}

the pump pulse energy, $w_{P}\left(w_{S}\right)$ is the pump (probe) beam waist radius, $\tau_{P}\left(\tau_{S}\right)$ is the temporal half-width of the pump (probe) pulse at the $1 / e^{2}$ intensity level, and $L$ is sample length.

The derivation of (1) accounts for the temporal and spatial overlap of the pump and probe fields [32]. It is assumed that the pump/probe fields have a Gaussian profile in both space and time and that the confocal parameters of both beams are longer than the sample length so that a plane-wave approximation can be used inside the sample under study. Accordingly, lens (6) was chosen such that the confocal parameter of the beams within the crystal was $>60 \mathrm{~mm}$, i.e. longer than the KGW $(30 \mathrm{~mm})$ and diamond $(6.5 \mathrm{~mm})$ crystals.

The accuracy of this approach was first assessed by making measurements on KGW - a widely used and wellcharacterised Raman crystal. The pump and probe beams propagated along the $30 \mathrm{~mm}$ length of the crystal and hence parallel to the $N_{p}$ axis (Fig. 1 (c)). Measurements were made for pump and probe beams co-polarised along the $N_{m}$ axis (to measure the gain at $901 \mathrm{~cm}^{-1}$ Raman shift) and the along the $N_{g}$ axis $\left(767 \mathrm{~cm}^{-1}\right.$ Raman shift) [24] (Fig. 4). The corresponding Raman gain coefficients were calculated with equation (1) using the values of the pump/probe beam waist radii of $600 / 100 \mu \mathrm{m}$ and the pump/probe pulse temporal halfwidths of $20 / 20 \mathrm{~ns}$ (at the $1 / e^{2}$ point in both cases). These were deduced from Gaussian fits to the measured spatial and temporal profiles. The Raman gain coefficients were calculated to be $5.7 \pm 0.5 \mathrm{~cm} / \mathrm{GW}$ for $767 \mathrm{~cm}^{-1}$ Raman shift and of $5.2 \pm 0.5 \mathrm{~cm} / \mathrm{GW}$ for $901 \mathrm{~cm}^{-1}$ Raman shift. This is in good agreement with previously reported values (Table 2 ).

The Raman gain in the diamond was measured using sample B (Fig. 1 (b)) for two directions of pump and probe propagation - along a $<100>$ direction ( $2 \mathrm{~mm}$ path length) and a $<110>$ direction (6.5 $\mathrm{mm}$ path length). The pump/probe beam waist radii were measured to be $400 / 180 \mu \mathrm{m}$, and the 


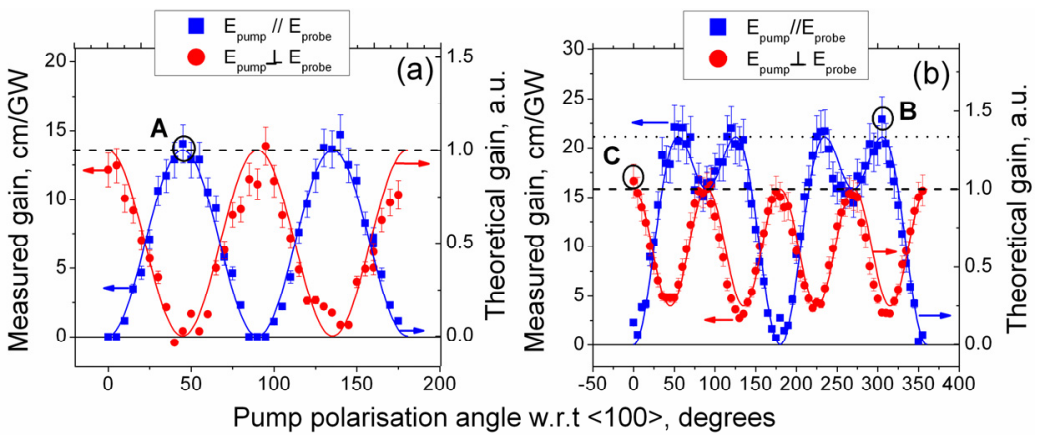

Fig. 5: Raman gain coefficient as a function of pump polarisation angle for pump and probe propagation along (a) the $<100>$ axis and (b) the $<110>$ axis in diamond. Measurements were made with the polarisation of the probe both parallel and perpendicular to that of the pump.

pump/probe pulse temporal half widths were measured to be 20/20 ns. The actual pump pulse energy inside the diamond was calculated to be $97 \%$ of the incident pulse energy. This takes account of both the loss of energy due to the Fresnel reflection from the front surface of the diamond and, at the same time, additional energy in the crystal originating the Fresnel reflection from the rear side of the diamond. During measurements, the diamond was tilted by $1^{\circ}$ with respect to the pump axis to avoid any resonance of Stokes emission inside the crystal. There was also a wedge of $0.9^{\circ}$ between the end faces, further reducing the likelihood of resonance.

Both theory [35, 36] and recent experimental results [17] indicate that Raman gain in diamond is dependent on both the propagation direction and the polarisation of the pump and probe with respect to the crystallographic axes. Therefore, the relative Raman gain in diamond for propagation along both $<100>$ and $<110>$ directions was measured as a function of crystal orientation with respect to the pump and probe beam polarisation. In Fig. 5 (a), the variation in the Raman gain with the angle of the pump polarisation with respect to a $<100>$ direction is plotted for propagation along a $<100>$ direction. Two data sets are shown: one for the probe polarisation parallel to that of the pump (squares) and a second where the polarisations are perpendicular (circles). Similar data is plotted in Fig. 5 (b), but for propagation along a $<110>$ direction.

The left-hand axes in Fig. 5 (a) and (b) were then calibrated in terms of the absolute Raman gain coefficient by directly measuring the maximum Raman gain of the diamond. This was first done for propagation along a $<100>$ direction with the pump and probe co-polarised along a $<110>$ direction (i.e. $45^{\circ}$ with respect to a $<100>$ direction) - corresponding to the point $\mathrm{A}$ in Fig. 5 (a). Measurements were then made for propagation along a $<110>$ direction: first with pump and probe co-polarised along a $<111>$ direction (i.e. $54.7^{\circ}$ with respect to a $<100>$ direction) - corresponding to the point $\mathrm{B}$ in Fig. 5 (b); and second with the pump and probe polarisations perpendicular and the pump polarised along a $<100>$ direction - corresponding to the point $\mathrm{C}$ in Fig. 5 (b). The results of those measurements are presented in Fig. 6. The maximum Raman gain coefficient for propagation along a $<100>$ direction was measured to be $14 \pm 2 \mathrm{~cm} / \mathrm{GW}$ (Fig. 6 (a)) - this occurred with the pump and probe co-polarised along a $<110>$ direction (corresponding to point A on Fig. 5 (a)).

In line with the theory presented below, and as observed by Sabella et al. [17] in measurements of Raman laser threshold, the gain in $<110>$-cut diamond, when the pump and probe are co-polarised along a $<111>$ direction (i.e. at $54.7^{\circ}$ to a $<100>$ direction), should be $33 \%$ higher than if the pump and probe polarisations are perpendicular and the pump polarisation is along $<100>$ direction. This improvement in the Raman gain is confirmed experimentally via the measurements of the Raman gain coefficient corresponding to the points $\mathrm{B}$ and $\mathrm{C}$ in Fig. 5 (b), presented in Fig. 6 (b).

The plots in Fig. 5 also include theoretical curves (solid lines) based on the equation in [35] for the Raman scattering efficiency, $S$, which is proportional to the Raman gain coefficient:

$S \propto\left|e_{S} R_{1} e_{P}\right|^{2}+\left|e_{S} R_{2} e_{P}\right|^{2}+\left|e_{S} R_{3} e_{P}\right|^{2}$

where $e_{P}$ and $e_{S}$ are the unit vectors of the pump and Stokes fields, respectively, and $R_{1-3}$ are the Raman polarisability tensors for diamond transformed to the laser illumination reference frame [36]. The $R_{1-3}$ tensors propagation along a $<100>$ direction in diamond are [35]:

$$
R_{1}=\left(\begin{array}{lll}
0 & 0 & 0 \\
0 & 0 & 1 \\
0 & 1 & 0
\end{array}\right) \quad R_{2}=\left(\begin{array}{lll}
0 & 0 & 1 \\
0 & 0 & 0 \\
1 & 0 & 0
\end{array}\right) \quad R_{3}=\left(\begin{array}{lll}
0 & 1 & 0 \\
1 & 0 & 0 \\
0 & 0 & 0
\end{array}\right)
$$

and for propagation along a $<110>$ direction are:

$$
\begin{aligned}
& R_{1}=\left(\begin{array}{ccc}
0 & 0 & 0 \\
0 & 1 & 0 \\
0 & 0 & -1
\end{array}\right) R_{2}=\left(\begin{array}{ccc}
0 & \sqrt{2} / 2 & \sqrt{2} / 2 \\
\sqrt{2} / 2 & 0 & 0 \\
\sqrt{2} / 2 & 0 & 0
\end{array}\right) \\
& R_{3}=\left(\begin{array}{ccc}
0 & \sqrt{2} / 2 & -\sqrt{2} / 2 \\
\sqrt{2} / 2 & 0 & 0 \\
-\sqrt{2} / 2 & 0 & 0
\end{array}\right)
\end{aligned}
$$

In Fig. 5, the predictions from equation (2) are shown on the right hand axes, the scaling of which has been adjusted such that the form of the dependence can be compared directly 

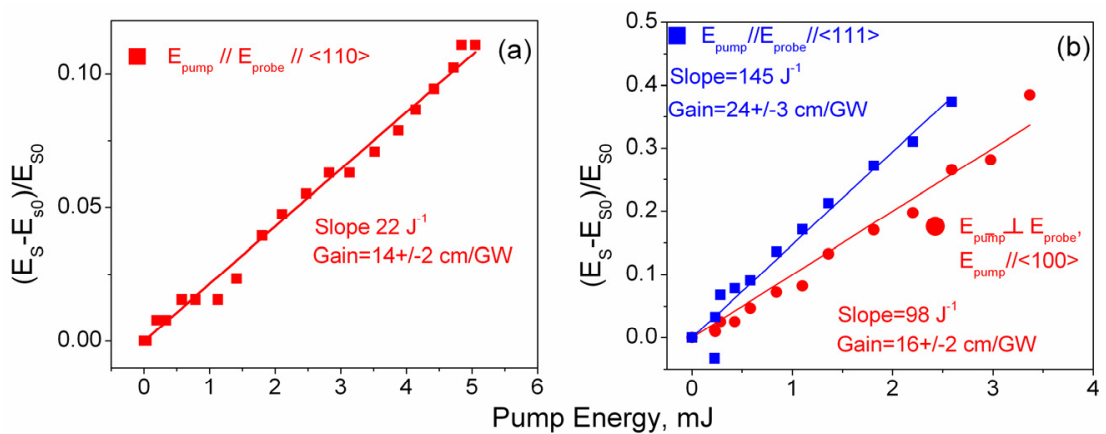

Fig. 6: Slopes of the Raman gain in diamond for (a) propagation along $<100>$ (2mm path length) and (b) propagation $<110>$ (6.5mm path length). Measurements are made with the polarisation of the probe both parallel and perpendicular to that of the pump.

with the experimental data. For propagation along a $<100>$ direction (Fig. 5 (a)), the Raman gain is a simple oscillatory function of the pump polarisation angle. The maximum value of the gain coefficient is estimated to be $14 \pm 2 \mathrm{~cm} / \mathrm{GW}$. This corresponds to a normalised Raman scattering efficiency of 1 as calculated by equation (2) (dash line in Fig. 5 (a)). Where the pump and probe are orthogonally polarised, the maximum gain is predicted and observed when both pump and probe are polarised along orthogonal $<100>$ directions. Where the pump and probe are co-polarised, the maximum gain occurs when both polarisations are at $45^{\circ}$ to a $<100>$ direction - that is to say along a $<110>$ direction.

For propagation along a $<110>$ direction when the pump and probe are orthogonally polarised (circles in Fig. 5 (b)), the maximum gain is estimated to be $15.5 \pm 2 \mathrm{~cm} / \mathrm{GW}$. As for the case of $\langle 100\rangle$ oriented diamond, this corresponds to a normalised Raman scattering efficiency of 1 (dash line in Fig. 5 (b)) as calculated by equation (2). Indeed, within the experimental error of $10 \%$, there is good agreement with the value of $14 \pm 2 \mathrm{~cm} / \mathrm{GW}$ estimated for the $<100>$ orientation.

For propagation along $\langle 110\rangle$ with the pump and probe orthogonally polarised, the dependence of the Raman gain on pump polarisation angle is a simple oscillatory function (circles in Fig. 5 (b)). Unlike the case for propagation along $<100>$, however, the Raman gain is never zero. If the pump and probe are co-polarised, the dependence on pump polarisation angle is more complicated. The measured Raman gain is maximised when the pump and probe are co-polarised along a $<111>$ direction - that is to say at $54.7^{\circ}$ to the $<100>$ direction. The value of the maximum gain in this case corresponds to a normalised Raman scattering efficiency of 1.33 (dotted line in Fig. 5 (b)) and the Raman gain coefficient is estimated to be $21 \pm 2 \mathrm{~cm} / \mathrm{GW}$ based on the best fit to the data. When the pump and probe are polarised along a $<110\rangle$ direction, the Raman gain coefficient drops to $15.5 \pm 2 \mathrm{~cm} / \mathrm{GW}$ (corresponding to a normalised Raman scattering efficiency of 1). The ratio of the Raman gain coefficient for polarisations along $<111\rangle$ and along $\langle 110\rangle$ agrees with the theoretically predicted 1.33:1 to within the experimental error.

To the authors' knowledge, there is only one previous report on the Raman gain coefficient in single-crystal CVD diamond at $1064 \mathrm{~nm}$ [37]. The Raman gain coefficient was reported to be $>12.5 \mathrm{~cm} / \mathrm{GW}$. This was estimated based on comparison of the stimulated Raman scattering threshold with that of another material with known Raman gain coefficient. However, it should be noted that the Raman gain coefficient of the latter was also estimated based on threshold comparison with the third Raman material [38].

Having experimentally confirmed the predictions of (2), it can be used to calculate - for a given propagation direction the maximum Raman scattering efficiency that can be achieved for a given pump polarisation angle, as well as the polarisation angle of the Raman scattered light at which this occurs. The results of such calculations for propagation along a $<100>$ direction are given in Fig. 7. The maximum Raman scattering efficiency - and therefore the maximum Raman gain - is constant in this case regardless of the pump polarisation angle; the minimum Raman gain is also constant and equal to zero (Fig. 7 (a)). The polarisation of the Raman scattered light at which the Raman scattering efficiency is maximised varies linearly with the pump polarisation such that the two polarisations are orthogonal when the pump is polarised along a $<100>$ direction and parallel when the pump is polarised along a $<110>$ direction (Fig. 7 (b)). These calculations indicate that, in the absence of other effects, the output polarisation of a diamond Raman laser will be linear where propagation is along a $<100>$ direction and that whilst the Raman gain will not change with the orientation of the pump polarisation, the Raman laser polarisation will vary with a dependence as shown in Fig. 7 (b).

The situation is again more complicated for propagation along a $<110>$ direction (Fig. 8). In this case, the maximum and minimum Raman scattering efficiencies predicted by (2) do depend on the pump polarisation angle, and the minimum Raman scattering efficiency is in general non-zero. The absolute maximum value of the Raman scattering efficiency is $33 \%$ higher than that of the $<100>$-cut diamond, in accordance with [17] and the experimental data presented above (Fig. 5 (b)). Moreover, Fig. 8(a) indicates that the minimum Raman scattering efficiency is zero only if the pump is polarised along a $<100>$ direction. For this scenario, the output polarisation of a Raman laser can be expected to be linearly polarised under all circumstances; however, for all other orientations of the pump, finite Raman gain on a polarisation orthogonal to that giving the maximum Raman gain would lead to the potential for the output polarisation to 

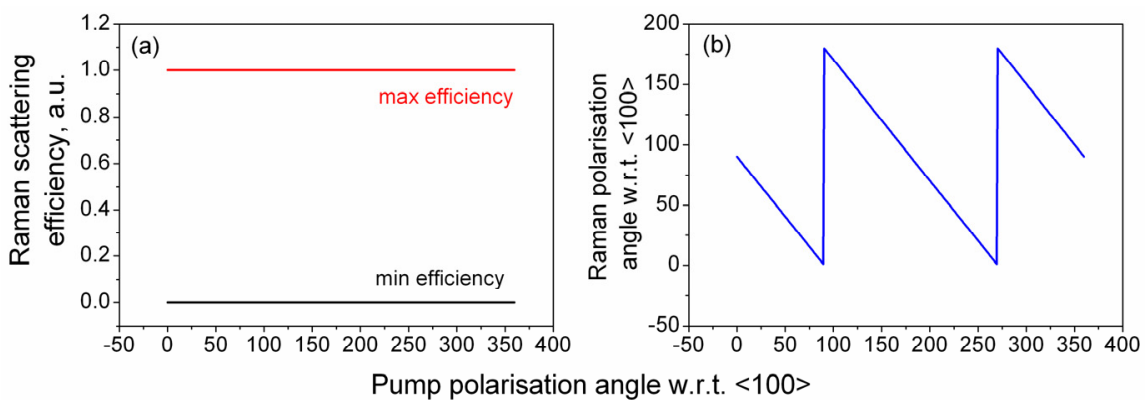

Fig. 7: (a) The maximum and minimum Raman scattering efficiency and (b) the polarisation of the Raman scattered light at which the Raman scattering efficiency is maximised, both as a function of the pump polarisation angle and for propagation along $<100>$. The polarisation angles are referenced to a $<100>$ direction.

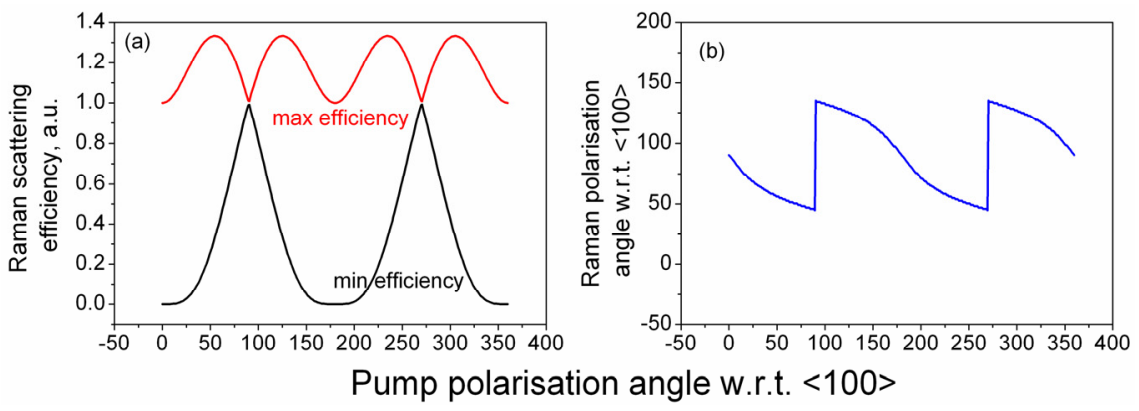

Fig. 8: (a) The maximum and minimum Raman scattering efficiency and (b) the polarisation of the Raman scattered light at which the Raman scattering efficiency is maximised, both as a function of the pump polarisation angle and for propagation along $<110>$. The polarisation angles are referenced to a $<100>$ direction

be other than linear. Indeed, where the pump is polarised along a $<110>$ direction (i.e. $90^{\circ}$ from the $<100>$ direction), the gain is equal for two orthogonal Raman laser polarisations. This is in agreement with the experimental observations reported in [17]. The angle of polarisation of the Raman output (with respect to a $<100>$ direction) at which the Raman gain is maximised, is shown in Fig. 8 (b). This indicates that when the pump polarisation is along a $<111>$ direction (i.e. at $54.7^{\circ}$ with respect to a $<100>$ direction), the pump will be copolarised with the Raman output and, at the same time, the Raman gain will have its absolute maximum value. This is in agreement with the measurements shown in Fig. 5 (b).

\section{CALORIMETRIC MEASUREMENTS OF LOSS}

An important consideration for intracavity use is the insertion loss of a material. For diamond, this is typically dominated by absorption associated with nitrogen impurities predominantly single substitutional nitrogen [21]. The absorption of early generations of single-crystal chemical vapour deposition grown diamond was measured by Turri et al. using laser calorimetry [27]. The absorption coefficients at $1064 \mathrm{~nm}$ ranged from 0.003 to $0.07 \mathrm{~cm}^{-1}$. However, much of the material investigated in this study - including the samples with the lowest absorption - had significant spatially varying birefringence. As van Loon et al. demonstrated [26], this birefringence made intracavity use of such material problematic. In 2010, Lubeigt et al. reported on the use of low-birefringence material $\left(\Delta n<5 \times 10^{-7}\right)$ to demonstrate the first continuous-wave diamond Raman laser [18]. However this material had an absorption coefficient of $\sim 0.03 \mathrm{~cm}^{-1}$ at 1064nm (inferred from Caird analysis of the intracavity losses [39]). This elevated loss limited the performance of the Raman laser. Subsequently, Friel et al. reported on the growth of single-crystal diamond that combined low birefringence $\left(\Delta n<10^{-6}\right)$ with an absorption coefficient at $1064 \mathrm{~nm}$ measured to be $\sim 0.001 \mathrm{~cm}^{-1}$ by ISO-standard laser calorimetry [21]. Material of this grade was then used to demonstrate an eight fold improvement in the output power of continuous wave diamond Raman lasers [11]. This indicates the importance of understanding the absorption characteristics of diamond if the performance of intracavity Raman lasers is to be optimised.

The absorption coefficient of the sample used here was measured using an adapted form of laser calorimetry. The voltage drop across a Peltier element due to the heat deposited by laser illumination was measured. The measurement set-up is presented in Fig. 9 (a). The sample under study (1) was placed on a $5.8 \times 3.8 \mathrm{~mm}$ Peltier element (2). This, in turn, was attached to a brass heat sink (3) using silver-loaded paint. Thermal grease was used to ensure a proper thermal contact between the sample and the Peltier element. A 3W laser beam at $1064 \mathrm{~nm}$ was incident on the sample under test. The laser beam (4) was focused into the sample by a lens (5). After passing through the sample, the beam was dissipated by a beam dump (6). The sample, Peltier element and the brass mount were covered with a styrofoam box (7) to ensure thermal isolation of the set-up. (N.B. In this configuration, it is not possible to distinguish between light absorbed directly in the sample and any small fraction scattered by the sample and subsequently absorbed in the Peltier element. Thus, a higher value of the absorption coefficient is to be expected from this 
(a)
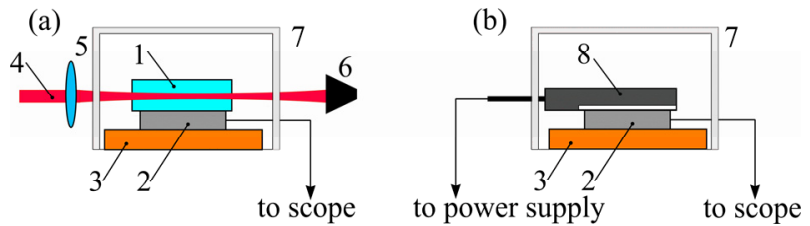

Fig. 9: Schematic diagram of the set-up used for the calorimetric measurements of loss (a), and for calibration of the set-up (b)

simplified technique compared to ISO-standard calorimetry.)

In order to calibrate the voltage drop across the Peltier element (Fig. 9 (b)), the sample was replaced with a resistor (8) (10 $\Omega$ thick-film power resistor (MP915) with the heat dissipated through a $5 \times 6.2 \mathrm{~mm}$ surface). The voltage drop across the Peltier element as function of the electrical power supplied to the resistor was measured giving a calibration coefficient $C=5.6 \mu \mathrm{W} / \mu \mathrm{V}$ assuming that all the electrical power was dissipated as heat in the resistor. The absorption coefficient $\alpha$ of the sample was then calculated using the equation:

$$
\alpha=-\frac{\ln (C \Delta U / P)}{L}
$$

where $L$ is the length of the sample, $\Delta U$ is the voltage drop across the Peltier element and $P$ is the laser power entering the sample under test.

The absorption coefficients at $1064 \mathrm{~nm}$ for the diamond sample A (Fig. 1 (a)) was measured to be $\sim 0.004 \mathrm{~cm}^{-1}$. This corresponds to a round trip loss of $0.5 \%$ given the sample lengths of $6.5 \mathrm{~mm}$. For comparative purposes, calorimetric absorption measurements were made on a $5 \mathrm{~mm}$ long undoped $3 \times 3 \mathrm{~mm}$ YAG sample (Molecular technology, MT-Berlin). The absorption coefficient was measured to be $0.001 \mathrm{~cm}^{-1}$. The manufacturer's specification for the absorption coefficient of the KGW sample used in this work was $<0.004 \mathrm{~cm}^{-1}$. As the absorption measurements for diamond sample A and for the samples in $[11,21]$ show, the absorption loss of modern synthetic diamond can now be of the same order as that of more conventional optical materials.

\section{CONTINUOUS-WAVE RAMAN LASERS RESUltS}

\section{A. Nd:YLF pump laser}

A high-power $\mathrm{Nd}: \mathrm{YLiF}_{4}$ (YLF) side-pumped laser module (manufactured by the Northrop-Grumman) was used to provide the $1 \mu \mathrm{m}$ laser radiation for subsequent Raman conversion. The 0.9at.\% Nd:YLF rod had dimensions of $\varnothing 3 \times 63 \mathrm{~mm}$. The end faces of the rod were antireflection coated at $1 \mu \mathrm{m}$. The module was water-cooled with a water temperature of $20^{\circ} \mathrm{C}$.

With the Nd:YLF module in a two mirror cavity, the maximum output power was $18.4 \mathrm{~W}$ for $153 \mathrm{~W}$ of incident laser diode pump power and the slope efficiency was $18 \%$. (N.B. the incident diode laser pump power is based on the manufacturer's calibration of the Nd:YLF laser head. The actual diode laser pump power cannot be measured in-situ and may be slightly lower due to diode ageing.) The cavity configuration was as recommended by the manufacturer for maximum output power: a curved mirror, with the radius of curvature (ROC) of $700 \mathrm{~mm}$ (highly-reflective (HR) at $1 \mu \mathrm{m}$ ) and a flat output coupler (OC) with the reflectivity (R) of $80 \%$ at $1 \mu \mathrm{m}$. The cavity length was $170 \mathrm{~mm}$. The output had a wavelength of $1047 \mathrm{~nm}$ and $\mathrm{M}^{2}$ parameters of $14 \times 19$ in the horizontal and vertical planes, respectively.

\section{B. Continuous-wave diamond Raman laser}

To enable intracavity pumping of the diamond Raman laser (Fig. 10), the cavities of the Nd:YLF laser and the diamond Raman laser were coupled using a dichroic mirror (DM: highly reflective at $1.1-1.25 \mu \mathrm{m}$, highly transmissive at $1 \mu \mathrm{m}$ ). This had the dual advantages of isolating the diamond Raman laser both from the thermal lens and the losses associated with the Nd:YLF rod. The cavity of the Nd:YLF laser was formed by the mirrors M1 to M4 (Fig. 10) which were highly reflective at $1 \mu \mathrm{m}$. The diamond Raman laser cavity was formed by the dichroic mirror (DM) and mirrors M2 to M4, with M2 and M3 being highly reflective at $1.2 \mu \mathrm{m}$. M4 was an output coupler for the Raman laser with a reflectivity of $1 \%$ at $1.2 \mu \mathrm{m}$. The design of the Nd:YLF cavity was stable against a thermal lens in the Nd:YLF rod with a focal length of $-750 \mathrm{~mm}$ (the value provided by the manufacturer). Assuming such a thermal lens, the waist radii of the fundamental mode of the $\mathrm{Nd}$ :YLF laser inside the Nd:YLF rod and the diamond were calculated to be 387 and $28 \mu \mathrm{m}$, respectively. The waist radius of the fundamental mode of the diamond Raman laser inside the diamond crystal was calculated to be $37 \mu \mathrm{m}$. The diamond crystal was wrapped in indium foil and mounted in a watercooled brass mount. The cooling water temperature was $15^{\circ} \mathrm{C}$.

Diamond sample A was orientated to exploit its $6.5 \mathrm{~mm}$ length $-\mathrm{a}<110>$ direction. The Nd:YLF laser polarisation was oriented along a $<100>$ axis of the diamond and the Raman polarisation was linear and parallel to a $<110>$ direction. Unfortunately, damage to the anti-reflection coatings on the diamond meant Raman laser oscillation with the Nd:YLF laser polarisation along a $<111>$ direction in diamond was not achieved. Improved performance can reasonably be expected when the samples are re-coated given the higher Raman gain available along $<111>$.

The maximum output power from the diamond Raman laser at $1217 \mathrm{~nm}$ was measured to be $5.1 \mathrm{~W}$ at $153 \mathrm{~W}$ of incident laser diode pump power (Fig. 11). The conversion efficiency of the fundamental laser emission to the Raman laser wavelength was $28 \%$, calculated as the ratio of the maximum output power of the diamond Raman laser to that of the $\mathrm{Nd}$ :YLF laser in the two-mirror cavity configuration discussed above under the same pumping conditions. The maximum slope efficiency of the diamond Raman laser was estimated to be $9.1 \%$ with respect to the diode laser pump power supplied to the Nd:YLF laser. The output power of the Raman laser was stable to within $10 \%$ of the mean value and was mainly determined by the noise on the fundamental emission at $1 \mu \mathrm{m}$ 


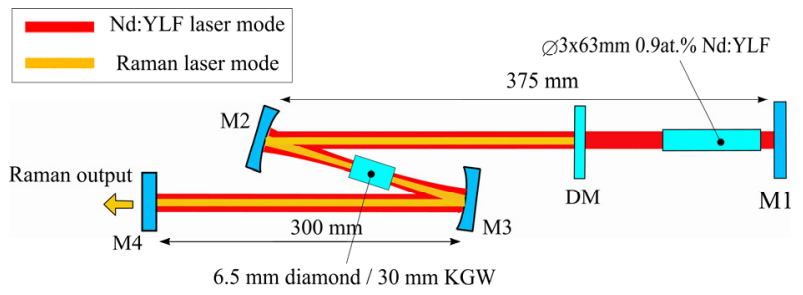

Fig. 10: Schematic diagram of the Raman laser. M1 - flat, HR at $1047 \mathrm{~nm}$; DM - flat dichroic mirror, HR at $1.1-1.2 \mu \mathrm{m}$, HT at $1 \mu \mathrm{m}$; M2 $\mathrm{ROC}=100 \mathrm{~mm}, \mathrm{HR}$ at $1-1.26 \mu \mathrm{m} ; \mathrm{M} 3-\mathrm{ROC}=100 \mathrm{~mm}, \mathrm{HR}$ at 1 and $1.2 \mu \mathrm{m}$ (for the diamond) and $\mathrm{HR}$ at $1-1.15 \mu \mathrm{m}$ (for the $\mathrm{KGW}$ ); M4 flat, $\mathrm{HR}$ at $1 \mu \mathrm{m}, \mathrm{R}=99 \%$ at $1.2 \mu \mathrm{m}$ (for the diamond) and $\mathrm{R} \sim 99.6 \%$ at $1139 \mathrm{~nm}$ (for the KGW). The distances between the curved mirrors M2 and $\mathrm{M} 3$ are $108 \mathrm{~mm}$ (for the diamond) and $118 \mathrm{~mm}$ (for the KGW); the distance between the mirror M2 and the DM is $275 \mathrm{~mm}$.

from the Nd:YLF laser which was of the same order in the two mirror cavity configuration (with no Raman conversion).

The $\mathrm{M}^{2}$ beam propagation factor for the diamond Raman laser was measured to be $1.1 \times 1.2$ along the horizontal and vertical planes, respectively. The $\mathrm{M}^{2}$ of the residual fundamental beam of the Nd:YLF laser, measured through mirror M1, was $8 \times 30$. So-called 'Raman beam cleanup' has been observed and analysed before for Raman amplifiers [40, 41] and lasers [42] and can lead to significant enhancement of the brightness of the Raman laser beam in comparison with that of the fundamental laser. In this case, a 48-fold enhancement is observed comparing the diamond Raman laser to the two mirror Nd:YLF laser. The brightness $(B)$ of the laser beam was calculated using the following equation [43]:

$$
B=P /\left(\lambda^{2} M^{2}{ }_{x} M^{2}{ }_{y}\right)
$$

where $P$ is the laser output power, $\lambda$ is the laser wavelength, $M_{x}^{2}$ and $M_{y}^{2}$ are the $\mathrm{M}^{2}$ factors of the laser beam along horizontal and vertical planes. No roll-over in the Raman laser output power was detected, suggesting there are good prospect for further power scaling using a more powerful pump source. In an attempt to study the effect of increased output coupling, the $1 \%$ output coupler was replaced by a $2.5 \%$ output coupler; however, Raman laser oscillation was not achieved.

\section{Continuous-wave $K G W$ Raman laser}

The same basic cavity design as for the diamond Raman laser was exploited. The distance between the mirrors M2 and M3 (Fig. 10) was increased from 108 to $118 \mathrm{~mm}$ to compensate for insertion of a longer Raman crystal. The KGW crystal (Fig. 1 (c)) was wrapped in indium foil and mounted in a water-cooled brass mount. The cooling water temperature was $15^{\circ} \mathrm{C}$.

Unfortunately, some of the available mirrors - designed for diamond Raman lasers operating between 1215 and 1240nm were not ideal for KGW Raman lasers with the output wavelengths between 1140-1155nm (corresponding to the KGW Raman shifts of 768 and $\left.901 \mathrm{~cm}^{-1}\right)$. Mirror M3 was therefore replaced with a mirror coated for high reflectivity between 1 and $1.15 \mu \mathrm{m}$, but this still introduced some

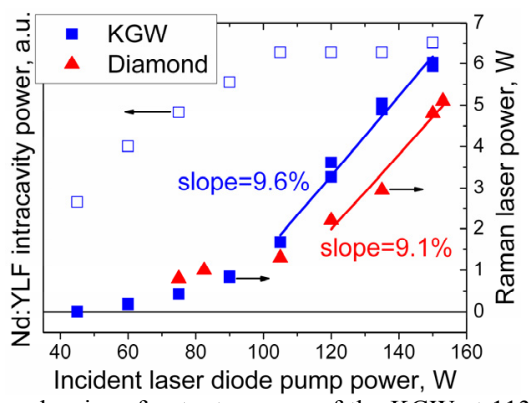

Fig. 11: Dependencies of output powers of the KGW at $1139 \mathrm{~nm}$ (solid squares) and diamond at $1217 \mathrm{~nm}$ (triangles) Raman lasers on the incident laser diode pump power. The intracavity power of the Nd:YLF laser during operation of the KGW Raman laser is also shown (open squares)

additional losses at the KGW Raman laser output wavelengths (mirror M2 had a broader reflectivity spectrum with negligible losses at the KGW Raman wavelength). The polarisation of the Nd:YLF laser was along the $N_{g}$ axis of the KGW crystal in order to gain access to the $768 \mathrm{~cm}^{-1}$ Raman shift. In this case, the Raman laser wavelength was expected to be $1139 \mathrm{~nm}$, giving lower losses at M3 than if the $901 \mathrm{~cm}^{-1}$ shift had been used. With these precautions, the maximum output power through M3 and M4, a total output coupling of $\sim 1 \%$ - was $6.1 \mathrm{~W}$ for $150 \mathrm{~W}$ of incident diode laser pump power.

In order to produce the output in a single beam, an alternative coupled cavity was designed (Fig. 2). Here the $\mathrm{Nd}$ :YLF laser cavity was formed by three mirrors M1 to M3, all highly reflective at $1047 \mathrm{~nm}$. The KGW Raman laser cavity was formed by the output coupler M4 (reflectivity $99.2 \%$ at $1139 \mathrm{~nm}$ ), dichroic mirror DM and two curved mirrors M2 and M3, the latter exhibited negligible output coupling at $1139 \mathrm{~nm}$ at normal incidence. Assuming a $-750 \mathrm{~mm}$ focal length thermal lens in the Nd:YLF rod, the waist radii of the fundamental mode of the Nd:YLF laser inside the Nd:YLF rod and the $\mathrm{KGW}$ crystal were calculated to be 382 and $28 \mu \mathrm{m}$, respectively. The waist radius of the fundamental mode of the KGW Raman laser inside the KGW crystal was calculated to be $40 \mu \mathrm{m}$. With this cavity design, the maximum output power was $6.03 \mathrm{~W}$ at $150 \mathrm{~W}$ of incident diode laser pump power (Fig. 11). This equates to a fundamental to Raman wavelength conversion efficiency of $33 \%$. The maximum slope efficiency of the Raman laser output power was estimated to be $9.6 \%$ with respect to the diode laser pump power supplied to the Nd:YLF rod. The output power was stable to within $10 \%$ of the mean value and, as for the diamond Raman laser, this was mainly determined by the noise of the Nd:YLF laser.

For both the diamond and KGW Raman lasers, the slope efficiency reaches its maximum value for diode-laser pump powers above about 100W (Fig. 1). Above this point, the intracavity field at the fundamental wavelength (open squares in Fig. 1 for the case of the KGW Raman laser) increases much more slowly with diode-laser pump power. This near clamping of the intracavity fundamental field is in line with theoretical predictions [12], but the behaviour between the Raman laser threshold and $\sim 100 \mathrm{~W}$ diode laser pump power where the intracavity power at the fundamental increases 


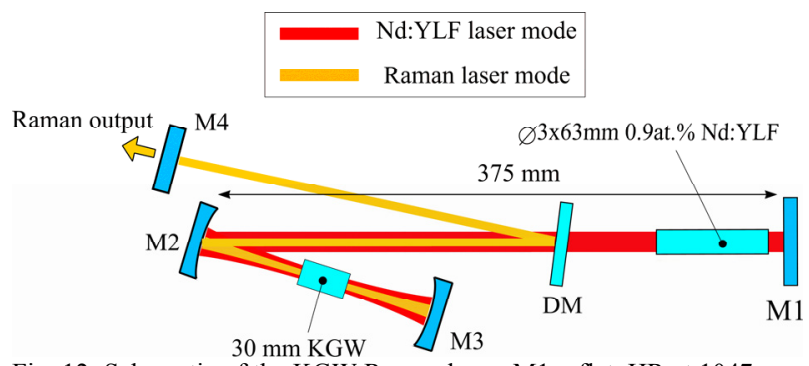

Fig. 12: Schematic of the KGW Raman laser: M1 - flat, HR at 1047 nm; $\mathrm{DM}$ - flat dichroic mirror, HR at 1.1-1.2 $\mu \mathrm{m}$, HT at $1 \mu \mathrm{m}$; M2 $\mathrm{ROC}=100 \mathrm{~mm}, \mathrm{HR}$ at $1-1.26 \mu \mathrm{m} ; \mathrm{M} 3-\mathrm{ROC}=50 \mathrm{~mm}, \mathrm{HR}$ at $1-1.15 \mu \mathrm{m}$; M4 - flat, HR at $1 \mu \mathrm{m}, \mathrm{R}=99.2 \%$ at $1139 \mathrm{~nm}$. The distance between the curved mirrors M2 and M3 is $118 \mathrm{~mm}$. The distance between M4 and $\mathrm{M} 2$ is $300 \mathrm{~mm}$

much more rapidly - requires further investigation. It may be that this behaviour relates to the multi-transverse mode nature of the fundamental laser mode. The thresholds of the diamond and KGW Raman lasers (determined as the intercept of the linear fit lines in Fig. 11 with the horizontal axis) were estimated to be 100 and $85 \mathrm{~W}$ of incident diode pump power respectively. This is to say $\sim 20 \%$ higher for diamond Raman laser. This is thought to be due in part to the lower product of the Raman gain coefficient and length $(g L)$ for the diamond crystal $\left(\sim 105 \mathrm{~cm}^{2} / \mathrm{GW}\right)$ compared to the $\mathrm{KGW}\left(\sim 170 \mathrm{~cm}^{2} / \mathrm{GW}\right)$ and in part to the higher losses in the diamond Raman laser compared to the KGW Raman laser $(2.1 \%$ and $1.9 \%$ respectively including the output coupling). Due to the sidepumping scheme of the Nd:YLF laser, its efficiency $(\sim 12 \%)$ is significantly lower than that of the end-pumped lasers, which in turn means that the slope efficiencies of the Raman lasers reported here are lower than for systems based on endpumped neodymium lasers. Nonetheless, higher conversion efficiencies with KGW and especially diamond are expected after careful optimisation of output coupling parameters and crystal lengths.

The $\mathrm{M}^{2}$ factor of the KGW Raman laser was much higher than that of the diamond Raman laser. It was measured to be $5 \times 6$ in horizontal and vertical planes respectively, for the laser with configuration pictured in Fig. 12. Therefore, although the KGW Raman laser produced higher output power, the improvement in brightness over the 2 mirror Nd:YLF laser at $1047 \mathrm{~nm}$ was much lower than for the diamond Raman laser 2.5 -fold compared to 43 -fold. The poorer beam quality of the KGW Raman laser compared with the diamond Raman laser is thought to result, at least in part, from stronger thermal aberrations in the KGW crystal. Further investigation is required to confirm this hypothesis. Using eq. (30) in [2] and the material parameters from the Table 1 , the thermal lens in the KGW crystal due to the quantum defect between the pump and Stokes photons was calculated to be $-23 \mathrm{~cm}(\sim 0.6 \mathrm{~W}$ of heat deposited $)$ and that in the diamond to be $1600 \mathrm{~cm}(\sim 0.8 \mathrm{~W}$ of heat deposited).

\section{CONCLUSIONS}

In conclusion, the first multi-watt truly continuous wave diamond Raman laser has been demonstrated. This laser gave an output power of $5.1 \mathrm{~W}$ at $1217 \mathrm{~nm}$ with near diffraction limited beam quality. The conversion efficiency with respect to the optimised fundamental laser at $1047 \mathrm{~nm}$ was $28 \%$ and the brightness enhancement was 43-fold. Under the same pumping conditions, the KGW Raman laser produced a higher output power of $6.1 \mathrm{~W}$ with corresponding conversion efficiency of $33 \%$, but with significantly poorer beam quality leading to a brightness enhancement of only 2.5 -fold.

In addition, the Raman gain coefficient of diamond was measured using a pump-probe approach at a pump wavelength of $1064 \mathrm{~nm}$. Measurements were made for propagation along both a $<100>$ and a $<110>$ crystallographic direction in single-crystal synthetic diamond. The maximum Raman gain coefficient at the pump wavelength of $1064 \mathrm{~nm}$ was found to be $21 \pm 2 \mathrm{~cm} / \mathrm{GW}$ - this was for propagation along a $<110>$ direction with the pump and Stokes fields co-polarised along a $<111>$ direction and is some four fold higher than the Raman gain coefficient measured in KGW using the same technique.

These results - coupled with the recent advent of highoptical quality synthetic diamond - demonstrate the potential of synthetic diamond for high-power, high-brightness continuous-wave Raman lasers at wavelengths of interest for applications in medicine and bio-photonics.

\section{REFERENCES}

[1] M. H. Dunn and M. Ebrahimzadeh, "Parametric generation of tunable light from continuous-wave to femtosecond pulses," Science, vol. 286, pp. 1513-1517, 1999.

[2] H. M. Pask, "The design and operation of solid-state Raman lasers," Progress in Quantum Electronics, vol. 27, pp. 3-56, 2003.

[3] J. A. Piper and H. M. Pask, "Crystalline Raman lasers," IEEE Journal of Selected Topics in Quantum Electronics, vol. 13, pp. 692-704, 2007.

[4] P. Cerny, H. Jelinkova, P. G. Zverev, and T. T. Basiev, "Solid state lasers with Raman frequency conversion," Progress in Quantum Electronics, vol. 28, pp. 113-143, 2004.

[5] E. J. Woodbury and W. K. Ng, "Ruby Laser Operation in near IR," Proceedings of the Institute of Radio Engineers, vol. 50, pp. 2367, 1962.

[6] H. M. Pask, "Continuous-wave, all-solid-state, intracavity Raman laser," Optics Letters, vol. 30, pp. 2454-2456, 2005.

[7] A. A. Demidovich, A. S. Grabtchikov, V. A. Lisinetskii, V. N. Burakevich, V. A. Orlovich, and W. Kiefer, "Continuous-wave Raman generation in a diode-pumped $\mathrm{Nd}^{3+}: \mathrm{KGd}\left(\mathrm{WO}_{4}\right)_{2}$ laser," Optics Letters, vol. 30, pp. 1701-1703, 2005.

[8] L. Fan, Y.-X. Fan, Y.-Q. Li, H. Zhang, Q. Wang, J. Wang, and H.-T. Wang, "High-efficiency continuous-wave Raman conversion with a $\mathrm{BaWO}_{4}$ Raman crystal," Optics Letters, vol. 34, pp. 1687-1689, 2009.

[9] A. J. Lee, D. J. Spence, J. A. Piper, and H. M. Pask, "A wavelengthversatile, continuous-wave, self-Raman solid-state laser operating in the visible," Optics Express, vol. 18, pp. 20013-20018, 2010.

[10] A. J. Lee, H. M. Pask, D. J. Spence, and J. A. Piper, "Efficient $5.3 \mathrm{~W} \mathrm{cw}$ laser at $559 \mathrm{~nm}$ by intracavity frequency summation of fundamental and first-Stokes wavelengths in a self-Raman $\mathrm{Nd}: \mathrm{GdVO}_{4}$ laser," Optics Letters, vol. 35, pp. 682-684, 2010.

[11] W. Lubeigt, V. G. Savitski, G. M. Bonner, S. L. Geoghegan, I. Friel, J. E. Hastie, M. D. Dawson, D. Burns, and A. J. Kemp, "1.6W continuouswave Raman laser using low-loss synthetic diamond," Optics Express, vol. 19, pp. 6938-6944, 2011.

[12] J. K. Brasseur, P. A. Roos, K. S. Repasky, and J. L. Carlsten, "Characterization of a continuous-wave Raman laser in $\mathrm{H}_{2}$, " Journal of the Optical Society of America B-Optical Physics, vol. 16, pp. 1305$1312,1999$.

[13] A. S. Grabtchikov, V. A. Lisinetskii, V. A. Orlovich, M. Schmitt, R. Maksimenka, and W. Kiefer, "Multimode pumped continuous-wave solid-state Raman laser," Optics Letters, vol. 29, pp. 2524-2526, 2004. 
[14] O. Kitzler, A. McKay, and R. P. Mildren, "CW diamond laser architecture for high power Raman beam conversion (Postdeadline)," presented at Conference on Lasers and Electro Optics Pacific Rim, Sydney, 2011.

[15] R. P. Mildren, J. E. Butler, and J. R. Rabeau, "CVD-diamond external cavity Raman laser at 573 nm," Optics Express, vol. 16, pp. 1895018955, 2008.

[16] J.-P. M. Feve, K. E. Shortoff, M. J. Bohn, and J. K. Brasseur, "High average power diamond Raman laser," Optics Express, vol. 19, pp. 913 922, 2011.

[17] A. Sabella, J. A. Piper, and R. P. Mildren, "1240 nm diamond Raman laser operating near the quantum limit," Optics Letters, vol. 35, pp. 3874 3876,2010

[18] W. Lubeigt, G. M. Bonner, J. E. Hastie, M. D. Dawson, D. Burns, and A. J. Kemp, "Continuous-wave diamond Raman laser," Optics Letters, vol. 35, pp. 2994-2996, 2010.

[19] R. S. Balmer, J. R. Brandon, S. L. Clewes, H. K. Dhillon, J. M. Dodson, I. Friel, P. N. Inglis, T. D. Madgwick, M. L. Markham, T. P. Mollart, N Perkins, G. A. Scarsbrook, D. J. Twitchen, A. J. Whitehead, J. J. Wilman, and S. M. Woollard, "Chemical vapour deposition synthetic diamond: materials, technology and applications," Journal of PhysicsCondensed Matter, vol. 21, pp. 364221, 2009.

[20] I. Friel, S. L. Clewes, H. K. Dhillon, N. Perkins, D. J. Twitchen, and G. A. Scarsbrook, "Control of surface and bulk crystalline quality in single crystal diamond grown by chemical vapour deposition," Diamond and Related Materials, vol. 18, pp. 808-815, 2009.

[21] I. Friel, S. L. Geoghegan, D. J. Twitchen, and G. A. Scarsbrook, "Development of high quality single crystal diamond for novel laser applications," Proceedings of SPIE, vol. 7838, p. 783819, 2010.

[22] I. V. Mochalov, "Laser and nonlinear properties of the potassium gadolinium tungstate laser crystal $\mathrm{KGd}\left(\mathrm{WO}_{4}\right)_{2}: \mathrm{Nd}^{3+}$ (KGW:Nd)," Optical Engineering, vol. 36, pp. 1660-1669, 1997.

[23] P. A. Loiko, K. V. Yumashev, N. V. Kuleshov, G. E. Rachkovskaya, and A. A. Pavlyuk, "Thermo-optic dispersion formulas for monoclinic double tungstates $\mathrm{KRe}\left(\mathrm{WO}_{4}\right)_{2}$ where $\mathrm{Re}=\mathrm{Gd}, \mathrm{Y}, \mathrm{Lu}, \mathrm{Yb}, "$ Optical Materials vol. 33, pp. 1688-1694, 2011.

[24] T. T. Basiev, V. N. Voĭtsekhovskiĭ, P. G. Zverev, F. V. Karpushko, A. V. Lyubimov, S. B. Mirov, V. P. Morozov, I. V. Mochalov, A. A. Pavlyuk, G. V. Sinitsyn, and V. É. Yakobson, "Conversion of tunable radiation from a laser utilizing an $\mathrm{LiF}$ crystal containing $\mathrm{F}_{2}^{-}$color centers by stimulated Raman scattering in $\mathrm{Ba}\left(\mathrm{NO}_{3}\right)_{2}$ and $\mathrm{KGd}\left(\mathrm{WO}_{4}\right)_{2}$ crystals," Soviet Journal of Quantum Electronics, vol. 17, pp. 1560, 1987.

[25] A. R. Lang, "Causes of Birefringence in Diamond," Nature, vol. 213, pp. 248-251, 1967.

[26] F. van Loon, A. J. Kemp, A. J. Maclean, S. Calvez, J.-M. Hopkins, J. E. Hastie, M. D. Dawson, and D. Burns, "Intracavity diamond heatspreader in lasers: the effects of birefringence," Optics Express, vol. 14, pp. 92509260, 2006

[27] G. Turri, Y. Chen, M. Bass, D. Orchard, J. E. Butler, S. Magana, T. Feygelson, D. Thiel, K. Fourspring, R. V. Dewees, J. M. Bennett, J. Pentony, S. Hawkins, M. Baronowski, A. Guenthner, M. D. Seltzer, D. C. Harris, and C. M. Stickley, "Optical absorption, depolarization, and scatter of epitaxial single-crystal chemical-vapor-deposited diamond at 1.064 um," Optical Engineering, vol. 46, pp. 064002, 2007.

[28] P. Millar, R. B. Birch, A. J. Kemp, and D. Burns, "Synthetic Diamond for Intracavity Thermal Management in Compact Solid-State Lasers," IEEE Journal of Quantum Electronics vol. 44, pp. 709-717, 2008.

[29] A. J. Maclean, R. B. Birch, P. W. Roth, A. J. Kemp, and D. Burns, "Limits on efficiency and power scaling in semiconductor disk laser with diamond heatspreaders," Journal of the Optical Society of America B-Optical Physics, vol. 26, pp. 2228-2236, 2009.

[30] J. J. Ottusch and D. A. Rockwell, "Measurement of Raman Gain Coefficients of Hydrogen, Deuterium, and Methane," IEEE Journal of Quantum Electronics, vol. 24, pp. 2076-2080, 1988.

[31] V. A. Lisinetskii, S. V. Rozhok, D. N. Bus'ko, R. V. Chulkov, A. S. Grabtchikov, V. A. Orlovich, T. T. Basiev, and P. G. Zverev, "Measurements of Raman gain coefficient for barium tungstate crystal," Laser Physics Letters, vol. 2, pp. 396-400, 2005.

[32] R. Stegeman, C. Rivero, G. Stegeman, P. Delfyett, K. Richardson, L. Jankovic, and H. Kim, "Raman gain measurements in bulk glass samples," Journal of the Optical Society of America B-Optical Physics, vol. 22, pp. 1861-1867, 2005.
[33] V. A. Berenberg, S. N. Karpukhin, and I. V. Mochalov, "Stimulated Raman scattering of nanosecond pulses in a $\mathrm{KGd}\left(\mathrm{WO}_{4}\right)_{2}$ crystal," Soviet Journal of Quantum Electronics, vol. 17, pp. 1178, 1987.

[34] P. G. Zverev, T. T. Basiev, A. A. Sobol, V. V. Skornyakov, L. I. Ivleva, N. M. Polozkov, and V. V. Osiko, "Stimulated Raman scattering in alkaline-earth tungstate crystals," Quantum Electronics, vol. 30, pp. 5559,2000 .

[35] R. Loudon, "The Raman effect in crystals," Advances in Physics, vol. 13, pp. 423 - 482, 1964

[36] J. Mossbrucker and T. A. Grotjohn, "Determination of local crystal orientation of diamond using polarized Raman spectra," Diamond and Related Materials, vol. 5, pp. 1333-1343, 1996

[37]A. A. Kaminskii, R. J. Hemley, J. Lai, C. S. Yan, H. K. Mao, V. G. Ralchenko, H. J. Eichler, and H. Rhee, " High-order stimulated Raman scattering in CVD single crystal diamond," Laser Physics Letters, vol. 4, pp. 350-353, 2007

[38] A. A. Kaminskii, T. Kaino, T. Taima, A. Yokoo, M. Moczka, and G. M. A. Gad, "Monocrystalline 2-Adamantylamino-5-Nitropyridine (AANP) a Novel Organic Material for Laser Raman Converters in the Visible and Near-IR," Japanese Journal of Applied Physics, vol. 41, pp. L603-L605, 2002

[39] J. A. Caird, S. A. Payne, P. R. Staver, A. J. Ramponi, L. L. Chase, and W. F. Krupke, "Quantum Electronic-Properties of the $\mathrm{Na}_{3} \mathrm{Ga}_{2} \mathrm{Li}_{3} \mathrm{~F}_{12}: \mathrm{Cr}^{3+}$ Laser," IEEE Journal of Quantum Electronics, vol. 24, pp. 1077-1099, 1988.

[40] V. I. Bespalov, A. A. Betin, and G. A. Pasmanik, "Reproduction of the pumping wave in stimulated-scattering radiation," Radiophysics and Quantum Electronics, vol. 21, pp. 675-688, 1978.

[41] J. Reintjes, R. H. Lehmberg, R. S. F. Chang, M. T. Duignan, and G. Calame, "Beam Cleanup with Stimulated Raman-Scattering in the Intensity-Averaging Regime," Journal of the Optical Society of America B-Optical Physics, vol. 3, pp. 1408-1427, 1986.

[42] J. T. Murray, W. L. Austin, and R. C. Powell, "Intracavity Raman conversion and Raman beam cleanup," Optical Materials, vol. 11, pp. 353-371, 1999.

[43] R. Paschotta, Encyclopedia of Laser Physics and Technology: WileyVCH, 2008.

Vasili G. Savitski received the Engineer Diploma in optics from the Belarus National Technical University, Minsk, in 1999, and the Ph.D. degree from the Institute of Physics, Minsk, in 2005 for work on the nonlinear optics of nanoparticles in glasses. He worked at the International Laser Center, Minsk on the nonlinear optics of nanoparticles, passive mode locking and thermal effects in lasers from 1999 to 2007. Currently, he is with the Institute of Photonics, Glasgow, where he is working on diamond Raman lasers.

Ian Friel received the B.Sc. degree in Physics from the University of Nottingham, UK in 1995 and the Ph.D. degree from Boston University, US in 2005 for work on the growth and characterisation of III-nitride heterostructures by molecular beam epitaxy. He joined Element Six Ltd. in 2005 where he is a Senior Scientist. Ian's research involves the synthesis of CVD diamond for advanced applications such as optics and electronics. His work also encompasses fundamental studies of CVD diamond growth and defects.

Jennifer E. Hastie (M'00) received the Ph.D. degree from the University of Strathclyde, Glasgow, U.K., in 2003 for work on semiconductor disk lasers and was subsequently awarded a research fellowship by the Royal Academy of Engineering to develop of visible and ultraviolet semiconductor disk lasers for applications in biophotonics. She is an Associate Team Leader at the Institute of Photonics, University of Strathclyde, the holder of the Challenging Engineering award from the UK Engineering and Physical Science Research Council, and a member of the Young Academy of Scotland.

Martin D. Dawson (M'85-SM'98-F'09) received the B.Sc. degree in physics and the Ph.D. degree in laser physics from Imperial College London, London, U.K., in 1981 and 1985, respectively. His thesis covered optical gain switching in semiconductor lasers and simultaneous mode locking and Qswitching in Nd:YAG lasers. He was a Visiting Assistant Professor first at North Texas State University, Denton, and subsequently at the University of Iowa, Iowa City, from 1985 to 1991, working on the development of femtosecond dye lasers and applications to the spectroscopy of III-V 
semiconductors. From 1991 to 1996, he was a Senior Researcher at the Sharp Laboratories of Europe Ltd., Oxford, U.K., performing optical spectroscopy on AlGaAs and AlInGaP semiconductors. He is a Professor and is the Director of Research at the Institute of Photonics, University of Strathclyde, Glasgow, where he leads the III-V Semiconductor Optoelectronics Program. His research interests include semiconductor disk lasers, nitride optoelectronics, and microfabrication of optical materials. Prof. Dawson is a fellow of the U.K. Institute of Physics, the Optical Society of America, and the Royal Society of Edinburgh.

David Burns received the B.Sc. degree (Hons.) from the University of Glasgow, Glasgow, U.K., and the Ph.D. degree from the University of St. Andrews, St. Andrews, U.K., for work on semiconductor and fibre lasers. He is currently an Associate Director and Team Leader for solid-state laser development at the Institute of Photonics, University of Strathclyde, Glasgow. His research interests include optically pumped semiconductor lasers, laser stabilization techniques, adaptive optics and microelectromechanical systems for lasers, mid-IR lasers, and optical parametric oscillators.

Alan J. Kemp (M'07) received the B.Sc. degree (Hons.) from the University of Glasgow, Glasgow, U.K., in 1996, and the Ph.D. degree from the University of St. Andrews, St. Andrews, U.K., in 1999 for work on microchip lasers. He worked on ultrafast lasers at the University of St. Andrews from 1999 to 2002. In 2002, he moved to the Institute of Photonics, University of Strathclyde, Glasgow, where he works on the use of diamond in lasers. 